\title{
Synchronized Breathing with Pelvic Floor Contractions Expands Maximum Voluntary Ventilation in Young Soccer Players
}

\author{
Vijayamurugan Eswaramoorthi ${ }^{1}$, Mohamad Razali Abdullah, ${ }^{2,}$, Naresh Bhaskar Raj ${ }^{1}$, \\ Rabiu Muazu Musa ${ }^{3}$, Ahmad Bisyri Husin Musawi Maliki ${ }^{2}$ \\ ${ }^{1}$ Faculty of Health Science, School of Rehabilitation Science, UniSZA, Gong Badak Campus, Kuala Nerus, Malaysia \\ ${ }^{2}$ Faculty of Applied Social Science, UniSZA, Gong Badak Campus, Kuala Nerus, Malaysia \\ ${ }^{3}$ Centre for Fundamental and Continuing Education, Universiti Malaysia Terengganu, Kuala Nerus, Malaysia
}

Received November 5, 2020; Revised December 28, 2020; Accepted January 20, 2021

\section{Cite This Paper in the following Citation Styles}

(a): [1] Vijayamurugan Eswaramoorthi, Mohamad Razali Abdullah, Naresh Bhaskar Raj, Rabiu Muazu Musa, Ahmad Bisyri Husin Musawi Maliki , "Synchronized Breathing with Pelvic Floor Contractions Expands Maximum Voluntary Ventilation in Young Soccer Players," International Journal of Human Movement and Sports Sciences, Vol. 9, No. 1, pp. 149 - 155, 2021. DOI: 10.13189/saj.2021.090121.

(b): Vijayamurugan Eswaramoorthi, Mohamad Razali Abdullah, Naresh Bhaskar Raj, Rabiu Muazu Musa, Ahmad Bisyri Husin Musawi Maliki (2021). Synchronized Breathing with Pelvic Floor Contractions Expands Maximum Voluntary Ventilation in Young Soccer Players. International Journal of Human Movement and Sports Sciences, 9(1), 149 - 155. DOI: 10.13189/saj.2021.090121.

Copyright $\bigcirc 2021$ by authors, all rights reserved. Authors agree that this article remains permanently open access under the terms of the Creative Commons Attribution License 4.0 International License

\begin{abstract}
The intention of this investigation was to decide the most significant exercise intervention in enhancing maximum voluntary ventilation in young male soccer players. Sixty non-smoking young male soccer players with the mean age of $14.18(0.79)$ years, were randomly assigned to four groups (Group A, B, C, D) of 20 players in each group. Maximum voluntary ventilation was measured at baseline using digital pony FX micro spirometer. The Group A athletes were instructed to do diaphragmatic breathing exercise with conventional sports training. Group B athletes received pelvic floor muscle exercise with conventional sports training. Group C athletes received synchronized diaphragmatic breathing with pelvic floor contraction with conventional sports training and Group D (Control group) players received conventional sports training alone. The training protocol was designed for 15 minutes duration 5 times per week for 8 weeks, along with their 60 minutes conventional sports training. Mid and post intervention measurements were carried out at the fourth week and the eighth week with identical baseline assessment protocol. The repeated measures of ANOVA revealed that, marked improvement in maximum voluntary ventilation was evident amongst all
\end{abstract}

of intervention groups except control group however post hoc analyses implicated that synchronized diaphragmatic breathing with pelvic floor contraction was beneficial in enhancing maximum voluntary ventilation than the pelvic floor exercise, diaphragmatic breathing exercise and conventional sports training $(\mathrm{P}<.05)$. Therefore, synchronized diaphragmatic breathing with pelvic floor contraction training should be considered as a part of sports training to improve maximum voluntary ventilation.

Keywords Maximum Voluntary Ventilation, Soccer, Pelvic Floor Exercise, Diaphragmatic Breathing Exercise, Sports Training

\section{Introduction}

Association football (also known as soccer, particularly in North America) is generally familiar to be the world's greatest popular complex sport [1]. Great aerobic longevity is a key factor for success in soccer. Aerobic durability majorly depends on respiratory functions of a 
player [2].

Advanced methods to boost performance had concentrated on keeping well-conditioned lungs [3]. Breathing is a secret weapon in sports used by players which can make the change among winning and losing at game [4]. Wellness in breathing increases athletic efficiency ensures a runner can run longer and a swimmer can swim more quickly and a foot baller can play more easily. This dynamic breathing function wellness is identified by maximum voluntary ventilation test. This maximum breathing capacity or maximum voluntary ventilation can be measured using spirometer [5].

Maximal voluntary ventilation (MVV) is one of the breathing parameter utilised to decide respiratory muscle endurance. The usual strategy of quantifying MVV is to let the player breathe as quick and deep as possible for 12 seconds, then multiply the entire flow volume by 5 to get the complete volume of ventilation per minute (measured as $\mathrm{L} / \mathrm{min}$ ) [6].

Diaphragmatic breathing exercise is recommended to enrich breathing capacity and for effective performance in sports.[7]. Similarly, to enrich breathing capacity, recent research recommends pelvic floor muscle exercises [5]. Scientific evidence argues that the pelvic floor muscles contract and relax dynamically during every breath, similar to the diaphragm, they move caudally during inspiration cranially during expiration along with the diaphragm [8].

Even though the breathing capacity and the pulmonary functions are influenced by both the synchronised diaphragmatic breathing with pelvic floor muscle contractions, reviews exist on either diaphragmatic breathing exercise on breathing function or pelvic floor muscle exercise on breathing function.

So which intervention is best to enhance breathing capacity is still unclear. Meanwhile synchronized diaphragmatic breathing with pelvic floor contraction has been greatly unnoticed or not caught the attention of the researchers. So, investigation became essential for this synchronized diaphragmatic breathing with pelvic floor contraction, and its impact on maximum breathing capacity. Therefore, this study aims to investigate the most significant exercise intervention in enhancing maximum voluntary ventilation in young male soccer players.

\section{Materials and Methods}

\subsection{Participants}

With randomized control trial design, a total of 60 non-smoking young male soccer players with age range of 13-15 years, in the absence of any musculoskeletal, cardio-respiratory, neurological disorder and resided for a minimum of one year duration in Malaysia were enrolled for the trial based on the sample size calculation using $G$ power version (3.1). The players were from soccer team of Majlis Sukan Negeri Terengganu (MSNT), Malaysia. The players who got respiratory disturbance, major illness couldn't learn exercise and could not attend the training session properly were excluded from the study. The coaches and the players were educated about the purpose of the research. All players were requested to sign the informed consent form earlier to the study. The current trial was commenced after the approval of the Universiti Sultan Zainal Abdin human research ethical committee (UHREC/2018/01) following which the playes were brought to the exercise therapy laboratory, Faculty of Health Sciences, UniSZA for the baseline assessments.

\subsection{Anthropometric Measurements}

The height, weight and BMI of the players were measured and documented using stadiometer and weighting scale.

\subsection{Measurement of Maximum Voluntary Ventilation}

Maximum voluntary ventilation was determined using a digital Pony FX micro spirometer (Cosmed, Rome). The measurement of Maximum voluntary ventilation was done using the protocol presented in Han and На (2015); Eswaramoorthi et al., (2018). [5], [9]. Maximum voluntary ventilation test was completed while the players were sitting on a chair with their extremities and shoulders straight and legs spread shoulder-width apart and at right angles to the ground. They were taught to close their nose by the use of a nose clamp and hold the evaluating apparatus with one hand and let it nip the mouth. Then the players were led to complete inspiration and expiration for 12 seconds in the deepest and fastest manner. Parameter measurement was taken 3 times, and the average value was used in the analysis.

\subsection{Methodology}

The players were selected from a population based on cluster sampling. After the completion of the sampling randomization was done via Research Randomizer (Version 4.0) [Computer software] [10] to sort the players into four groups 1) Group A - Diaphragmatic exercise training group received diaphragmatic breathing exercise with conventional sports training $(\mathrm{n}=20)$; 2) Group B Pelvic floor muscle exercise training group received pelvic floor muscle exercise with conventional sports training ( $n=20)$; 3) Group $\mathrm{C}$-training group received synchronized diaphragmatic breathing with pelvic floor contraction with conventional sports training $(n=20)$ and 4) Group D - control group received conventional sports training alone $(\mathrm{n}=20)$. Mid and post intervention measurements were carried out at the fourth week and the 
eighth week with identical baseline assessment protocol.

\subsection{Diaphragmatic Breathing Exercise Training Parotocol}

A standard yoga mat was provided to each of the player during the exercise practice session. Then the players were asked to lie on yoga mat in a relaxed crook-lying position (lay supine with both hip joints flexed to 45 degrees and foot lying flat on the floor) with placing their hands on the upper part of the abdomen (immediately below the frontal costal cartilage), after that the players were instructed to inhale slowly and deeply through their nose mean while they were asked to concentrate on allowing their abdominal wall to raise gently and slowly with fixed upper chest and relaxed shoulder. Then they were told to exhale slowly in the controlled fashion through their mouth mean while again they were asked to concentrate on their abdomen wall to sinking slowly back to rest [11]. Assigned players were requested to do this exercise training for 15 minutes duration 5 times per week for 8 weeks, along with their conventional sports training [12].

\subsection{Pelvic Floor Muscle Exercise Training Protocol}

Initially the players were given training to identify the pelvic floor muscles correctly to do the exercise, and then a standard yoga mat was provided to each of the player during the Pelvic floor muscles exercise practice session. Then the players were asked to lie on yoga mat in a relaxed crook-lying position (Lay supine with both hip joints flexed to 45 degrees and foot lying flat on the floor with resting their arms by the side of the subject) without contracting the abdominal, lumbar, gluteal, and lower-limb muscles. Then the player was asked to contract the pelvic floor muscles alone for 5 seconds slowly, means at the end of fifth second, they should reach the maximum strength of contraction. Then at the sixth second, they were asked to relax, followed with fast contraction and relaxation between the seventh and ninth seconds, then they were asked to rest the muscle in the tenth second. While doing so the players was asked to maintain their normal breath and the breath holding was prohibited. One set of exercises included performing ten set of the above mentioned exercise. A 40-second break was provided between the sets. This protocol of pelvic floor exercise intervention was adapted in ref [5]. Assigned athletes were requested to do this exercise training for 15 minutes duration 5 times per week for 8 weeks, along with their conventional sports training.

\subsection{Synchronized Breathing with Pelvic Floor Contraction Training Protocol}

Phase-locked synchronized movement of diaphragm and pelvic floor during breathing was concentrated for this exercise training. Initially the players were given training to identify the pelvic floor muscles correctly to do the exercise, and then a standard yoga mat was provided to each of the players during the Synchronized Breathing with pelvic floor contraction training practice session. Then the players were asked to lie on yoga mat in a relaxed crook-lying position (Lay supine with both hip joints flexed to 45 degrees and foot lying flat on the floor) with placing their hands on the upper part of the abdomen (immediately below the frontal costal cartilage), after that the athletes were instructed to inhale slowly and deeply through their nose mean while they were asked to voluntarily relax their pelvic floor muscles till the end phase of inhalation. Then they were told to exhale slowly in the controlled fashion through their mouth mean while again they were asked to contract their pelvic floor muscles voluntarily as much as possible till the end phase of exhalation [8]. Assigned players were requested to do this exercise training for 15 minutes duration 5 times per week for 8 weeks, along with their conventional sports training.

\subsection{Conventional Sports Training Protocol}

The Conventional sports training followed in this study comprised of exercises in four categories which were commonly used by football coaches. This includes warm-up session, skill exercises, group activities (Mini football game), and cool-down stretching period. The sports exercise training was planned and supervised by football coaches using sports specific training protocol of Majlis Sukan Negeri Terengganu (MSNT), and the athletes also instructed to maintain a dairy about their exercise training. Blind periodical monitoring was done by the investigator on the spot. All athletes were instructed to follow their coaches for their sports training exercise for 60 minutes duration 5 times per week for 8 weeks.

\subsection{Statistical Analysis}

At the end of the 8th week the data of the final players after drop out was taken for analysis to find out the mean differences across groups at various times [17]-[19]. The data were analysed using SPSS version 24.0 replicated from previous researches [20]-[21]. Descriptive statistics and repeated measures ANOVA, within and between interactions was done to compute the results of the study following analysis.

\section{Results and Discussion}

The information was picked up from 60 players since there was drop out of around 20 players from the groups. The anthropometric characteristics of the players is shown in Table 1.60 young male soccer players took an interest in this investigation with, the mean age of 14 years 
[14.18(0.79)]; the mean BMI was $19 \mathrm{~kg} / \mathrm{m} 2$ [19.70(1.46)] with a mean height and weight of $158 \mathrm{~cm}$ [158.75(9.52)] and $49 \mathrm{~kg}$ [49.87(7.36)] respectively. Table 2 delineates the mean difference in maximum voluntary ventilation across different interventions and stages. It could be distinguished that the maximum voluntary ventilation improved across all the intervention groups aside from control group. The normality of the data was recognized by Kolmogorov-Smirnov test. Mauchly's test of Sphericity was done to assess the variances of difference among the groups followed by Greenhouse Geisser corrections due to the violation of the sphericity. Table 3 shows the comparison of the maximum voluntary ventilation with each treatment group based on time. There was significant improvement on the maximum voluntary ventilation $(\mathrm{P}<0.05)$ at $4^{\text {th }}$ week and the end of $8^{\text {th }}$ week. A repeated measure ANOVA within group analysis was applied followed by pairwise comparison with $95 \%$ confidence interval adjustment by Shefe correction. The outcomes show that there were significant differences in all intervention groups $(\mathrm{P}<0.05)$ apart from control group.

Table 1. Anthropometrics quality of the players

\begin{tabular}{|c|c|c|c|c|c|}
\hline Variables & $\begin{array}{c}\text { Over all } \\
\mathrm{n}=60 \\
\text { Mean (SD) }\end{array}$ & $\begin{array}{c}\text { Group A } \\
\mathrm{n}=15 \\
\text { Mean (SD) }\end{array}$ & $\begin{array}{c}\text { Group B } \\
\mathrm{n}=15 \\
\text { Mean (SD) }\end{array}$ & $\begin{array}{c}\text { Group C } \\
\mathrm{n}=15 \\
\text { Mean (SD) }\end{array}$ & $\begin{array}{c}\text { Group D } \\
\mathrm{n}=15 \\
\text { Mean (SD) }\end{array}$ \\
\hline Age (Years) & $14.18(0.79)$ & $14.33(0.72)$ & $14.13(0.83)$ & $14.07(0.88)$ & $14.20(0.77)$ \\
\hline Height $(\mathrm{cm})$ & $158.75(9.52)$ & $162.60(11.32)$ & $155.93(9.99)$ & $157.80(8.47)$ & $158.67(7.55)$ \\
\hline Weight $(\mathrm{kg})$ & $49.87(7.36)$ & $50.79(9.38)$ & $49.56(6.56)$ & $50.22(8.05)$ & $48.88(5.49)$ \\
\hline BMI $\left(\mathrm{kg} / \mathrm{m}^{2}\right)$ & $19.70(1.46)$ & $19.04(1.37)$ & $20.35(1.56)$ & $20.06(1.69)$ & $19.35(0.81)$ \\
\hline
\end{tabular}

$\mathrm{SD}=$ Standard deviation

Table 2. Mean difference in maximum voluntary ventilation (liters/min)

\begin{tabular}{|c|c|c|c|c|}
\hline PHASES & $\begin{array}{c}\text { GROUP A } \\
\text { Diaphragmatic Breathing } \\
\text { Exercise Training } \\
\text { Mean (SD) }\end{array}$ & $\begin{array}{c}\text { GROUP B } \\
\text { Pelvic Floor Muscle } \\
\text { Exercise Training } \\
\text { Mean (SD) }\end{array}$ & $\begin{array}{c}\text { GROUP C } \\
\text { Synchronized } \\
\text { Breathing } \\
\text { Mean (SD) }\end{array}$ & $\begin{array}{c}\text { GROUP D } \\
\text { Conventional Sports } \\
\text { Training } \\
\text { Mean (SD) }\end{array}$ \\
\hline PRE & $59.9(18.86)$ & $62.55(15.59)$ & $69.03(20.30)$ & $65.82(20.89)$ \\
\hline MID & $68.77(20.53)$ & $77.37(17.16)$ & $87.12(22.56)$ & $66.24(20.85)$ \\
\hline POST & $77.56(21.59)$ & $75.78(20.42)$ & $104.76(26.40)$ & $65.36(18.88)$ \\
\hline
\end{tabular}

Table 3. Comparison of Maximum voluntary ventilation within each treatment group based on time (Time effect)

\begin{tabular}{|c|c|c|c|c|c|c|c|c|}
\hline \multirow[t]{2}{*}{ Comparison } & \multicolumn{2}{|c|}{$\begin{array}{c}\text { Group A } \\
\text { Diaphragmatic } \\
\end{array}$} & \multicolumn{2}{|c|}{$\begin{array}{c}\text { Group B } \\
\text { Pelvic Floor Training } \\
\end{array}$} & \multicolumn{2}{|c|}{$\begin{array}{c}\text { Group C } \\
\text { Synchronized Breathing } \\
\end{array}$} & \multicolumn{2}{|c|}{ Group D Control } \\
\hline & MD (95\% CI) & $\mathrm{p}$-value & MD (95\% CI) & $\mathrm{p}$-value & MD (95\% CI) & $\mathrm{p}$-value & $\begin{array}{l}\text { MD }(95 \% \\
\text { CI })\end{array}$ & p-value \\
\hline Pre-Mid & $\begin{array}{c}-8.87 \\
(-13.05,-4.70)\end{array}$ & 0.00 & $\begin{array}{c}-14.82 \\
(-22.06,-7.58) \\
\end{array}$ & 0.00 & $\begin{array}{c}-18.09 \\
(-25.82,-10.37)\end{array}$ & 0.00 & $\begin{array}{c}-0.45 \\
(-2.91,2.01)\end{array}$ & 1.00 \\
\hline Pre-Post & $\begin{array}{c}-17.66 \\
(-25.22,-10.10)\end{array}$ & 0.00 & $\begin{array}{c}-24.54 \\
(-34.57,-14.51)\end{array}$ & 0.00 & $\begin{array}{c}-35.73 \\
(-48.21,-23.26)\end{array}$ & 0.00 & $\begin{array}{c}0.44 \\
(-2.39,3.27) \\
\end{array}$ & 1.00 \\
\hline Mid-Post & $\begin{array}{c}-8.79 \\
(-12.69,-4.89) \\
\end{array}$ & 0.00 & $\begin{array}{c}-9.72 \\
(-14.54,-4.90)\end{array}$ & 0.00 & $\begin{array}{c}-17.64 \\
(-23.67,-11.61) \\
\end{array}$ & 0.00 & $\begin{array}{c}0.89 \\
(-1.01,2.79) \\
\end{array}$ & 0.70 \\
\hline
\end{tabular}

Based on estimated marginal means

*. The mean difference (MD) is significant at the .05 level.

b. Adjustment for multiple comparisons: Bonferroni.

Table 4. Overall mean differences of Maximum voluntary ventilation among intervention groups (Intervention effect)

\begin{tabular}{|c|c|c|}
\hline Comparison & Mean difference (95\% CI) & p-value \\
\hline Diaphragmatic- Pelvic floor & $-6.93(-27.72,13.86)$ & 0.82 \\
\hline Diaphragmatic -Synchronized & $-18.23(-39.02,2.56)$ & 0.11 \\
\hline Diaphragmatic - Control & $2.94(-17.85,23.73)$ & 0.98 \\
\hline Pelvic floor- Synchronized & $-11.3(-32.09,9.49)$ & 0.49 \\
\hline Pelvic floor- Control & $9.87(-10.92,30.66)$ & 0.6 \\
\hline Synchronized - Control & $21.17(0.39,41.96)$ & 0.04 \\
\hline
\end{tabular}


Table 5. Comparison of the Maximum voluntary ventilation among four different treatment groups based on time (Time-treatment interaction)

\begin{tabular}{|c|c|c|c|}
\hline & \multirow{2}{*}{ Comparison } & Mean difference & \multirow{2}{*}{ p-value } \\
\hline & & $(95 \% \mathrm{CI})$ & \\
\hline \multirow{6}{*}{ PRE } & Diaphragmatic- Pelvic floor & $-2.66(-21.65,16.34)$ & 1 \\
\hline & Diaphragmatic -Synchronized & $-9.13(-28.13,9.86)$ & 1 \\
\hline & Diaphragmatic - Control & $-5.9(-24.89,13.09)$ & 1 \\
\hline & Pelvic floor- Synchronized & $-6.48(-25.47,12.51)$ & 1 \\
\hline & Pelvic floor- Control & $-3.24(-22.24,15.75)$ & 1 \\
\hline & Synchronized - Control & $3.23(-15.76,22.23)$ & 1 \\
\hline \multirow{6}{*}{ MID } & Diaphragmatic- Pelvic floor & $-8.6(-28.95,11.75)$ & 1 \\
\hline & Diaphragmatic -Synchronized & $-18.35(-38.7,2)$ & 0.1 \\
\hline & Diaphragmatic - Control & $2.53(-17.82,22.88)$ & 1 \\
\hline & Pelvic floor- Synchronized & $-9.75(-30.1,10.6)$ & 1 \\
\hline & Pelvic floor- Control & $11.13(-9.22,31.48)$ & 0.84 \\
\hline & Synchronized - Control & $20.88(0.53,41.23)$ & 0.04 \\
\hline \multirow{6}{*}{ POST } & Diaphragmatic- Pelvic floor & $-9.53(-31.51,12.45)$ & 1 \\
\hline & Diaphragmatic -Synchronized & $-27.21(-49.19,-5.23)$ & 0.08 \\
\hline & Diaphragmatic - Control & $12.2(-9.78,34.18)$ & 0.81 \\
\hline & Pelvic floor- Synchronized & $-17.67(-39.65,4.31)$ & 0.19 \\
\hline & Pelvic floor- Control & $21.73(-0.25,43.71)$ & 0.05 \\
\hline & Synchronized - Control & $39.41(17.43,61.39)$ & 0 \\
\hline
\end{tabular}

The results of the intervention effect (Table 4) after repeated measures Anova analysis followed by post hoc multiple comparisons using Shefe correction show that synchronized breathing with pelvic floor contractions group was significantly better than conventional sports training $(\mathrm{P}<0.05)$ whereas there was no significant difference amongst other groups. Pairwise comparison of group analysis based on time (Table 5) highlights the difference in the maximum voluntary ventilation values across the intervention groups. It is evident that the synchronized breathing with pelvic floor contractions group was superior to conventional sports training at the end of $4^{\text {th }}$ weeks of intervention and at the end of $8^{\text {th }}$ weeks again synchronized breathing with pelvic floor contractions group found to be significantly better in improving the maximum voluntary ventilation compared to that of conventional sports training $(\mathrm{P}<0.05)$.

Changes in maximum voluntary ventilation of group $\mathrm{A}$ with diaphragmatic breathing exercise point out that, at the end of the fourth week the mean maximum voluntary ventilation improved from 59.9 (18.86) liters to 68.77 (20.53) liters and the rise was 13.78 per cent. After the fourth week of intervention to the end of the eighth week, it rised from 68.77 (20.53) liters to 77.56 (21.59) liters was 12.01 per cent. The total increase in mean maximum voluntary ventilation from baseline to the end of eighth week with diaphragmatic breathing exercise was 25.69 per cent.

Variations in maximum voluntary ventilation of group
B with pelvic floor muscle exercise intervention suggest that, at the end of the fourth week, mean maximum voluntary ventilation increased from 62.55 (15.59) liters to 77.37 (17.16) liters, with an increase of 21.18 per cent. From the fourth week of intervention to the end of the eighth week, it decreases from 77.37 (17.16) liters to 75.78 (20.42) liters was $2.07 \%$. The overall increase in mean maximum voluntary ventilation from baseline to the end of eighth week with pelvic floor muscle exercise intervention was 19.12 per cent.

Fluctuations in maximum voluntary ventilation of group $\mathrm{C}$ with synchronized diaphragmatic breathing with pelvic floor contraction exercise intervention propose that, at the end of the fourth week, mean maximum voluntary ventilation improved from 69.03 (20.30) liters to 87.12 (22.56) liters, with an increase of 23.17 per cent. From the fourth week of intervention to the end of the eighth week, it rised from 87.12 (22.56) liters to 104.75 (26.40) liters, the percentage of increase was $18.38 \%$. The whole increase in maximum voluntary ventilation from baseline to the end of eighth week with synchronized diaphragmatic breathing with pelvic floor contraction exercise intervention was 41.11 percent.

Variations in maximum voluntary ventilation of group D with conventional sports training intervention validate that, at the end of the fourth week, mean maximum voluntary ventilation was rises from 65.82 (20.89) liters to 66.24 (20.85) liters, with an increase of 0.63 per cent. Commencing from the fourth week of intervention to the 
end of the eighth week, it declined from 66.24 (20.85) liters to 65.36 (18.88) liters, with fall of 1.33 per cent. The overall reduction in mean maximum voluntary ventilation since baseline to the end of eighth week with conventional sports training intervention was 0.70 per cent.

The outcomes of the maximum voluntary ventilation (MVV) evidentially specify that there was an overall improvement in the maximum voluntary ventilation (MVV) of the players across various stages of assessment between the different intervention groups aside from control group. An in-depth investigation of the comparison amongst the group across the interventions discovered that, for the period of mid-term intervention assessment phases, players of synchronized breathing with pelvic floor contractions training group (Group C) $(\mathrm{M}=$ 87.12, $\mathrm{SD}=22.56$ ) was witnessed to effectively increased the maximum breathing capacity compared of their counterparts in the conventional sports training group $(\mathrm{M}=66.24, \mathrm{SD}=20.85)(\mathrm{p}<.05)$, In the post intervention assessment, the maximum breathing capacity achieved by the players of the synchronized breathing with pelvic floor contractions training group (Group C) $(\mathrm{M}=104.76, \mathrm{SD}=$ 26.40) were more compared to that of the players in the conventional sports training group $(\mathrm{M}=65.36, \mathrm{SD}=18.88)$ $(\mathrm{p}<.01)$.

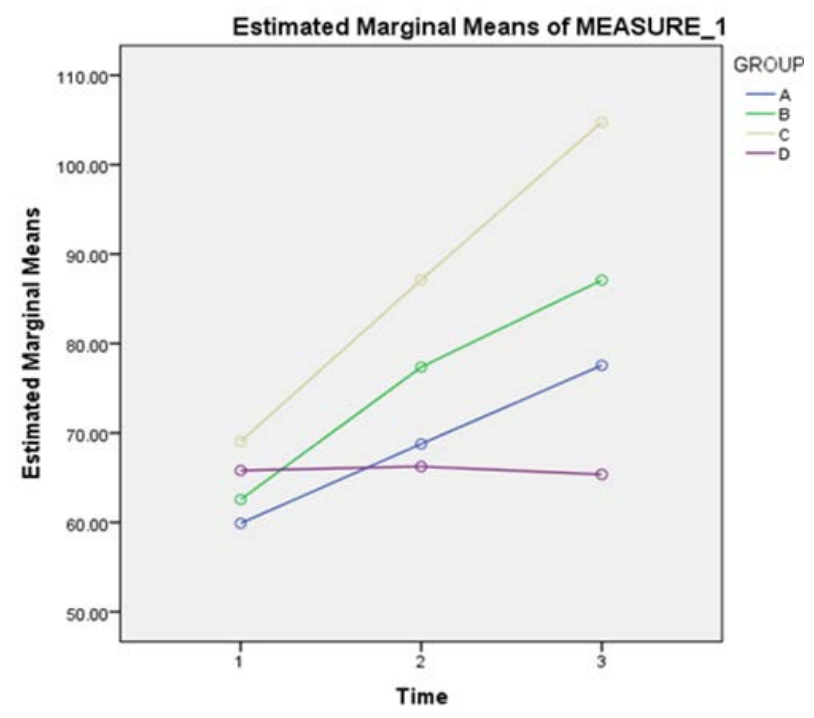

Figure 1. Profile plot of the mean maximum voluntary ventilation among four different groups based on time

Maximal voluntary ventilation (MVV) is in all the pulmonary function parameters accustomed to verify respiratory muscle endurance [13]. It is recognized that MVV relies on equally inspiratory and expiratory breathing effort: inspiratory air flow relies solely on inspiratory muscle power, but expiratory air flow depends chiefly on lung recoil. Lung recoil is understood to be the most important determinant of expiratory air flow in performing MVV [14]. Now the question may be raised as to why the players of synchronized breathing with pelvic floor contractions group (Group C) were evident as having highest maximum voluntary ventilation (MVV) compared to their counterparts from any other intervention groups, it could be postulated that this improvement was the outcome of greater improvement of respiratory and pelvic floor muscle strength, effective synchronisation diaphragm and pelvic floor contraction, and pulmonary capacity of those players who were subjected to synchronized breathing with pelvic floor contractions exercise training. Han \& Ha (2015) reported an increase in the maximum voluntary ventilation from $84.88 \mathrm{~L}$ to $112.62 \mathrm{~L}$ in reinforcement of the pelvic floor through pelvic floor exercise for 4 weeks, similarly the current study reported that maximum voluntary ventilation raised from $69.55 \mathrm{~L}$ to $104.78 \mathrm{~L}$ in the pelvic floor exercise training of 8 weeks [5]. Likewise, Yong, Lee \& Lee (2017) concluded diaphragmatic breathing exercise enhance respiratory function [15]. In addition, the respiratory muscles may be anticipated to progress respiratory functions through increasing muscular strength and endurance through various forms of exercise [16]. By enhancing ventilation through reinforcing strength and endurance of the respiratory muscles, respiratory imbalance is improved and tissue oxygenation is encouraged to boost daily life activities and quality of life. Here we would like to highlight that, both the previous researches considered pelvic floor exercise or diaphragmatic breathing exercise alone for pulmonary function improvement, whereas in this present study, synchronised intervention of diaphragmatic breathing with pelvic floor contractions was introduced, which has not yet been studied in any of the earlier researches and was suspected be a reason for improvement of maximum voluntary ventilation of players.

This study has a certain limitation Such as inclusion of male players alone, players from one state only were included, and measurement of parameters such muscular strength and sports performance changes were not included. Age matched controls also was not included in this study. These deficiencies should be well thought-out for forthcoming researches.

\section{Conclusion}

Diaphragmatic breathing, pelvic floor muscle exercise, synchronized breathing with pelvic floor contractions was effective in improving the maximum voluntary ventilation of young male soccer players. But synchronized breathing with pelvic floor contractions evidentially is verified to be better in enhancing the maximum voluntary ventilation of young male soccer players.

\section{Acknowledgements}

The authors honor the Research and Development Management Unit, University of Sultan Zainal Abidin 
(UniSZA), Kuala Terengganu for offering the internal research grant for the research (UniSZA/2016/DPU/17). The authors also convey their appreciation to Majlis Sukan Negeri Terengganu (MSNT), for their support in this study.

\section{REFERENCES}

[1] Giulianotti, R (2012) Football. The Wiley-Blackwell Encyclopedia of Globalization, New York.

[2] Birinci, M. C., Yılmaz A. K., Erkin, A., Sahbaz S and Aydın, İ. (2014) Determination of relationship between respiratory parameters and aerobic capacity of referees. Procedia-Social and Behavioral Sciences, 152, 353-357.

[3] Hakked, C. S., Balakrishnan, R., and Krishnamurthy, M. N. (2017). Yogic breathing practices improve lung functions of competitive young swimmers. Journal of Ayurveda and integrative medicine, 8(2), 99-104.

[4] Lomax, M., Grant, I., and Corbett, J. (2011). Inspiratory muscle warm-up and inspiratory muscle training: separate and combined effects on intermittent running to exhaustion. Journal of sports sciences, 29(6), 563-569.

[5] Han, D., and Ha, M. (2015). Effect of pelvic floor muscle exercises on pulmonary function. Journal of physical therapy science, 27(10), 3233-3235.

[6] Suh, M. R., Kim, D. H., Jung, J., Kim, B., Lee, J. W., Choi, W. A., and Kang, S. W. (2019). Clinical implication of maximal voluntary ventilation in myotonic muscular dystrophy. Medicine, 98(18), e15321.

[7] HajGhanbari, B., Yamabayashi, C., Buna, T. R., Coelho, J. D., Freedman, K. D., Morton, T. A., and Reid, W. D. (2013). Effects of respiratory muscle training on performance in athletes: a systematic review with meta-analyses. The Journal of Strength and Conditioning Research, 27(6), 1643-1663.

[8] Talasz, H., Kremser, C., Kofler, M., Kalchschmid, E., Lechleitner, M., and Rudisch, A. (2011). Phase-locked parallel movement of diaphragm and pelvic floor during breathing and coughing - a dynamic MRI investigation in healthy females. International urogynecology journal, 22(1), 61-68.

[9] Eswaramoorthi, V., Abdullah, M. R., Musa, R. M., Maliki, A. B. H. M., Kosni, N. A., Raj, N. B., and Juahir, H. (2018). A multivariate analysis of cardiopulmonary parameters in archery performance. Human Movement, 19(4), 35-41.

[10] Urbaniak G. C, Plous S. Research randomizer (version 4.0)[computer software]. 2013. Retrieved on: from http://www. randomizer. org/(aceessed June 22, 2020).
[11] Seo, K., Park, S. H., and Park, K. (2015). Effects of diaphragm respiration exercise on pulmonary function of male smokers in their twenties. Journal of Physical Therapy Science, 27(7), 2313-2315.

[12] Seo, K. C., Lee, H. M., and Kim, H. A. (2013). The effects of combination of inspiratory diaphragm exercise and exspiratory pursed-lip breathing exercise on pulmonary functions of stroke patients. Journal of Physical Therapy Science, 25(3), 241-244.

[13] Kor, A. C., Ong, K. C., Earnest, A., and Wang, Y. T. (2004). Prediction of the maximal voluntary ventilation in healthy adult Chinese subjects. Respirology, 9(1), 76-80.

[14] Lavietes, M. H., Clifford, E., Silverstein, D., Stier, F., and Reichman, L. B. (1979). Relationship of static respiratory muscle pressure and maximum voluntary ventilation in normal subjects. Respiration, 38(3), 121-126.

[15] Yong, M. S., Lee, H. Y., and Lee, Y. S. (2017). Effects of diaphragm breathing exercise and feedback breathing exercise on pulmonary function in healthy adults. Journal of physical therapy science, 29(1), 85-87.

[16] Carr, M. J., and Undem, B. J. (2003). Bronchopulmonary afferent nerves. Respirology, 8(3), 291-301.

[17] Zolkipli, H. M., Juahir, H., Adiana, G., Zainuddin, N., Ismail, A., Maliki, A. B. H. M., and Mokhtar, M. (2018). Spatial Assessment and the Most Significant Parameters for Drinking Water Quality Using Chemometric Technique: A Case Study at Malaysia Water Treatment Plants. International Journal of Engineering \& Technology, 7(3.14), $115-122$.

[18] Abu Hassan, M. S. N., Ismail, N. H., Abd Halim, N. S., Abd Aziz, M., Mohd Sidik, N. H., Kamarudin, M. K. A and Maliki, A. B. H. M. (2019). Research on male and female youth perceptions on the use of pornographic displays in social media. International Journal of Recent Technology and Engineering, 8(2), 284-287.

[19] Rozi, A. F. M., Abdullah, M. R., Rasid, S. M. M., Juahir, H., Maliki, A. B. H. M., Hashim, M. R., and Bidin, M. N. (2019). The development of Malaysian body somatotype model using comprehensive multivariate techniques. International Journal of Recent Technology and Engineering, 8(2), 264-267.

[20] Azahari, H., Abdullah, M. R., Maliki, A. B. H. M., Eswaramoorthi, V., Juahir, H., Musa, R. M., and Zawi, K. (2019). Physical Fitness and Anthropometric Characteristics in School Children: A Comparison of Urban and Rural Areas in East Coast of Peninsular Malaysia. Indian Journal of Public Health Research \& Development, 10(3), 619-623.

[21] Maliki, A. B. H. M., Abdullah, M. R., Juahir, H., Abdullah, F., Abdullah, N. A. S., Musa, R. M., and Nasir, N. A. M. (2018). A multilateral modelling of Youth Soccer Performance Index (YSPI). In IOP Conference Series: Materials Science and Engineering, 342(1), 012057. 